A NNALES

UNIVERSITATIS MARIAE CURIE-SKŁODOWSKA

LUBLIN - POLONIA

VOL. LXVIII, NO. 2, 2014

SECTIO A

$51-58$

\title{
ANDRZEJ KRYCZKA
}

\section{Deviation from weak Banach-Saks property for countable direct sums}

\begin{abstract}
We introduce a seminorm for bounded linear operators between Banach spaces that shows the deviation from the weak Banach-Saks property. We prove that if $\left(X_{\nu}\right)$ is a sequence of Banach spaces and a Banach sequence lattice $E$ has the Banach-Saks property, then the deviation from the weak Banach-Saks property of an operator of a certain class between direct sums $E\left(X_{\nu}\right)$ is equal to the supremum of such deviations attained on the coordinates $X_{\nu}$. This is a quantitative version for operators of the result for the KötheBochner sequence spaces $E(X)$ that if $E$ has the Banach-Saks property, then $E(X)$ has the weak Banach-Saks property if and only if so has $X$.
\end{abstract}

1. Introduction. A Banach space $X$ is said to have the Banach-Saks (BS) property if every bounded sequence in $X$ contains a subsequence $\left(x_{n}\right)$ whose Cesàro means $\sum_{i=1}^{n} x_{i} / n$ converge in norm. Such a property was proved by Banach and Saks [1] for $L_{p}[0,1]$ spaces with $1<p<\infty$. The case $p=1$ was examined by Szlenk [14] who proved that every weakly convergent sequence in $L_{1}[0,1]$ contains a subsequence with strongly convergent Cesàro means. This variant of the BS property is considered also for operators (see [2]). A bounded linear operator $T$ between Banach spaces $X$ and $Y$ is said to have the weak Banach-Saks (WBS) property if every weakly null sequence $\left(x_{n}\right)$ in $X$ contains a subsequence $\left(x_{n}^{\prime}\right)$ such that $\left(T x_{n}^{\prime}\right)$ is Cesàro convergent in $Y$.

2010 Mathematics Subject Classification. 46A45, 46B42.

Key words and phrases. Weak Banach-Saks property, Köthe-Bochner space, direct sum. 
In this note, we focus on weakly null sequences which have no Cesàro convergent subsequences. Some quantitative information on the deviation from summability of such sequences is provided by Rosenthal's dichotomy [13]. Recall that every weakly null sequence in a Banach space $X$ contains a subsequence $\left(x_{n}\right)$ such that either all subsequences of $\left(x_{n}\right)$ are Cesàro convergent in norm to zero or no subsequence of $\left(x_{n}\right)$ is Cesàro convergent and then there is a number $\delta>0$ such that $\left\|\sum_{n \in A} c_{n} x_{n}\right\| \geq \delta \sum_{n \in A}\left|c_{n}\right|$ for all scalars $\left(c_{n}\right)$ and all subsets $A \subset \mathbb{N}$ with $|A| \leq 2^{k}, k \leq \min A$ and $k \in \mathbb{N}$, where $|A|$ is the number of elements of $A$.

Using Rosenthal's result, Partington [12] proved that a Banach space $X$ has the WBS property if and only if for all $\varepsilon>0$ and weakly null sequences $\left(x_{n}\right)$ in $X$ there exists a finite subset $A \subset \mathbb{N}$ such that $\left\|\sum_{n \in A} x_{n}\right\|<\varepsilon|A|$. This served to prove that the direct sums of Banach spaces, built on a Banach space with a hyperorthogonal basis and the BS property, preserve the WBS property.

Our generalization of Partington's result for direct sums goes in two directions: it has a quantitative character and concerns operators. We introduce a seminorm for operators which measures the deviation from the WBS property. We consider a certain class of operators acting between direct sums $E\left(X_{\nu}\right)$. In the main result, we show that the deviation from the WBS property of an operator is equal to the supremum of such deviations attained on the coordinates $X_{\nu}$, providing that a Banach sequence lattice $E$ has the Banach-Saks property. Our main tool in the proofs is a repeated averaging technique elaborated in $[7,8]$, and based on the spreading models of Brunel and Sucheston [3].

2. Preliminaries. A Banach space $E$ of real-valued functions on $\mathbb{N}=$ $\{1,2,3, \ldots\}$ with the natural partial order is called a Banach sequence lattice if, for every finite subset $A \subset \mathbb{N}$, the characteristic function $\chi_{A}$ of $A$ belongs to $E$, and if $x=(x(\nu)) \in E$ and $|y(\nu)| \leq|x(\nu)|$ for every $\nu \in \mathbb{N}$, then $y=(y(\nu)) \in E$ and $\|y\|_{E} \leq\|x\|_{E}$. The lattice $E$ is said to be regular (or $\sigma$-order continuous) if, for every sequence $\left(x_{n}\right)$ in $E$ with $x_{n} \downarrow 0$, it holds $\lim _{n \rightarrow \infty}\left\|x_{n}\right\|_{E}=0$.

A Banach sequence lattice is a particular case of a Köthe function space with the counting measure space on $\mathbb{N}$ (see [9], [10]). Thus the Köthe dual space $E^{\prime}$ of $E$ is the space of all real-valued sequences $(y(\nu))$ such that $(x(\nu) y(\nu)) \in l_{1}$ for every $(x(\nu)) \in E$. The norm in $E^{\prime}$ is given for every $y=(y(\nu))$ by

$$
\|y\|_{E^{\prime}}=\sup \left\{\sum_{\nu=1}^{\infty}|x(\nu) y(\nu)|:\|x\|_{E} \leq 1, x=(x(\nu))\right\} .
$$

If $E$ is regular, then the Köthe dual space $E^{\prime}$ is isometrically isomorphic to the dual space $E^{*}$ (see [10, p. 29]). 
Let $E$ be a Banach sequence lattice and $\left(X_{\nu}\right)$ a sequence of Banach spaces. By $E\left(X_{\nu}\right)$ we mean the Banach space of all sequences $x=(x(\nu))$ such that $x(\nu) \in X_{\nu}$ for every $\nu \in \mathbb{N}$ and $\left(\|x(\nu)\|_{X_{\nu}}\right) \in E$. The norm in $E\left(X_{\nu}\right)$ is given by

$$
\|x\|_{E\left(X_{\nu}\right)}=\left\|\left(\|x(\nu)\|_{X_{\nu}}\right)\right\|_{E} .
$$

If $X_{\nu}=X$ for all $\nu$, then $E(X)$ is called a Köthe-Bochner sequence space.

If $E$ is regular, then the dual space $\left(E\left(X_{\nu}\right)\right)^{*}$ is isometrically isomorphic to $E^{*}\left(X_{\nu}^{*}\right)$ (see $[11$, Proposition 3.1$\left.]\right)$. Using this fact, we can prove a counterpart of Lemma 1 of [5] without the separability assumption.

Lemma 1. Let $E$ be a regular Banach sequence lattice. If $x_{n}=\left(x_{n}(\nu)\right) \in$ $E\left(X_{\nu}\right)$ for all $n \in \mathbb{N}$ and $x_{n} \stackrel{w}{\rightarrow} 0$ in $E\left(X_{\nu}\right)$, then $x_{n}(\nu) \stackrel{w}{\rightarrow} 0$ in $X_{\nu}$ for every $\nu \in \mathbb{N}$.

Proof. Fix $k \in \mathbb{N}$ and let $x^{*} \in X_{k}^{*}$. Put $(f(\nu))=\left(0, \ldots, 0, x^{*}, 0, \ldots\right)$ with $x^{*}$ on $k$ th place. Clearly, $(f(\nu)) \in E^{*}\left(X_{\nu}^{*}\right)$. Let $\tau$ be the isometric isomorphism between $\left(E\left(X_{\nu}\right)\right)^{*}$ and $E^{*}\left(X_{\nu}^{*}\right)$ given by Proposition 3.1 of [11] (see also [6]). There exists $f=\tau^{-1}[(f(\nu))]$ in $\left(E\left(X_{\nu}\right)\right)^{*}$ such that $f(x)=\sum_{\nu=1}^{\infty}\langle x(\nu), f(\nu)\rangle$ for every $x=(x(\nu)) \in E\left(X_{\nu}\right)$. Then

$$
f\left(x_{n}\right)=\sum_{\nu=1}^{\infty}\left\langle x_{n}(\nu), f(\nu)\right\rangle=\left\langle x_{n}(k), f(k)\right\rangle=x^{*}\left(x_{n}(k)\right)
$$

Since $\lim _{n \rightarrow \infty} f\left(x_{n}\right)=0$ and $x^{*} \in X_{k}^{*}$ was arbitrary, $x_{n}(k) \stackrel{w}{\rightarrow} 0$ in $X_{k}$.

3. Results. The space of all bounded linear operators between Banach spaces $X$ and $Y$ we denote by $L(X, Y)$. For a sequence $\left(x_{n}\right)$ in a Banach space, we put

$$
\psi\left(x_{n}\right)=\inf \left\{\left\||A|^{-1} \sum_{n \in A} x_{n}\right\|:|A|<\infty\right\} .
$$

In our quantitative considerations, we will need a certain stability of $\psi$ with respect to repeated averaging of $\left(x_{n}\right)$. This can be achieved through the process of arithmetic averaging of $\left(x_{n}\right)$ on equipollent successive blocks. We say that $\left(y_{n}\right)$ is a sequence of successive arithmetic means ( $\left.\operatorname{sam}\right)$ for $\left(x_{n}\right)$ if there exist $m \in \mathbb{N}$ and a sequence of subsets $I_{n} \subset \mathbb{N}$ with $\max I_{n}<\min I_{n+1}$ and $\left|I_{n}\right|=m$ such that $y_{n}=\sum_{i \in I_{n}} x_{i} / m$ for all $n$. Clearly, $\psi\left(x_{n}\right) \leq \psi\left(y_{n}\right)$.

The next result is a part of Proposition 2.3 of [7], where the proof based on spreading models was given for a similar characteristics of a sequence related to the alternate signs Banach-Saks property. The proof for $\psi$ runs in much the same way. We include it for completeness.

Proposition 2. Let $\left(x_{n}\right)$ be a bounded sequence in a Banach space $X$. Then for every $\varepsilon>0$ there exists a sequence $\left(y_{n}\right)$ of sam for $\left(x_{n}\right)$ such that for 
all finite subsets $A \subset \mathbb{N}$,

$$
\left\||A|^{-1} \sum_{n \in A} y_{n}\right\| \leq \psi\left(y_{n}\right)+\varepsilon
$$

Proof. If $\left(x_{n}\right)$ contains a Cauchy subsequence $\left(x_{n}^{\prime}\right)$, it is enough to ignore a finite number of terms of $\left(x_{n}^{\prime}\right)$ and put $y_{n}=x_{n}^{\prime}$. Assume now that $\left(x_{n}\right)$ has no Cauchy subsequence. We follow in part the line of the proof of Theorem II.2 of [2]. We extract a subsequence $\left(x_{n}^{\prime}\right)$ of $\left(x_{n}\right)$ that is the fundamental sequence of the spreading model $F$ built on $\left(x_{n}\right)$. Put

$$
K=\inf \left\{\left\||A|^{-1} \sum_{n \in A} x_{n}^{\prime}\right\|_{F}:|A|<\infty\right\} .
$$

There exist a finite subset $I \subset \mathbb{N}$ and $z=\sum_{i \in I} x_{i}^{\prime} /|I|$ such that $K \leq$ $\|z\|_{F} \leq K+\varepsilon / 4$. Let $\left(I_{n}\right)$ be a sequence of subsets $I_{n} \subset \mathbb{N}$ with $\max I_{n}<$ $\min I_{n+1}$ and $\left|I_{n}\right|=|I|$ for all $n$. Put $z_{n}=\sum_{i \in I_{n}} x_{i}^{\prime} /\left|I_{n}\right|$. Since the norm of $F$ is invariant under spreading, $\left\|z_{n}\right\|_{F}=\|z\|_{F}$ for all $n$. Consequently, $K \leq\left\|\sum_{n \in A} z_{n} /|A|\right\|_{F} \leq K+\varepsilon / 4$ for all finite subsets $A \subset \mathbb{N}$.

By $\left[2\right.$, Proposition I.1], for every $k \in \mathbb{N}$, we can choose $n_{k}$ so that for all $A \subset \mathbb{N}$ with $|A| \leq 2^{k}$ and $n_{k} \leq \min A$,

$$
\left\||A|^{-1} \sum_{n \in A} z_{n}\right\|-\left\||A|^{-1} \sum_{n \in A} z_{n}\right\|_{F} \mid<\varepsilon / 4 .
$$

We may assume that $n_{k}<n_{k+1}$. Let $z_{k}^{\prime}=z_{n_{k}}$. Then for all $A \subset \mathbb{N}$ with $|A| \leq 2^{k}$ and $k \leq \min A$,

$$
K-\varepsilon / 4 \leq\left\||A|^{-1} \sum_{n \in A} z_{n}^{\prime}\right\| \leq K+\varepsilon / 2 .
$$

Passing to a sequence of the arithmetic means of $\left(z_{n}^{\prime}\right)$ built on long enough successive blocks, we show now similar estimates for all finite $A \subset \mathbb{N}$. Let $|A|<\infty$ and $A_{0}=\left\{n \in A: n<\log _{2}|A|\right\}$. Then

$$
\left\|\sum_{n \in A_{0}} z_{n}^{\prime}\right\| \leq\left|A_{0}\right|(K+\varepsilon / 2), \quad\left\|\sum_{n \in A \backslash A_{0}} z_{n}^{\prime}\right\| \geq\left(|A|-\left|A_{0}\right|\right)(K-\varepsilon / 4) .
$$

It follows that

$$
\begin{aligned}
\left\||A|^{-1} \sum_{n \in A} z_{n}^{\prime}\right\| & \geq|A|^{-1}\left(\left\|\sum_{n \in A \backslash A_{0}} z_{n}^{\prime}\right\|-\left\|\sum_{n \in A_{0}} z_{n}^{\prime}\right\|\right) \\
& \geq K-\varepsilon / 4-\left|A_{0}\right||A|^{-1}(2 K+\varepsilon / 4) .
\end{aligned}
$$


There exists $m \in \mathbb{N}$ such that if $|A| \geq m$, then $\left|A_{0}\right||A|^{-1}(2 K+\varepsilon / 4) \leq \varepsilon / 4$ and, consequently,

$$
K-\varepsilon / 2 \leq\left\||A|^{-1} \sum_{n \in A} z_{n}^{\prime}\right\|<K+\varepsilon / 2 .
$$

Put $y_{n}=\sum_{i \in J_{n}} z_{i}^{\prime} /\left|J_{n}\right|$, where $\left(J_{n}\right)$ is a sequence of subsets $J_{n} \subset \mathbb{N}$ with $\max J_{n}<\min J_{n+1}$ and $\left|J_{n}\right|=m$ for all $n$. Then

$$
\left\||A|^{-1} \sum_{n \in A} y_{n}\right\| \leq \psi\left(y_{n}\right)+\varepsilon
$$

for every finite $A \subset \mathbb{N}$. Clearly, $\left(y_{n}\right)$ is a sequence of sam for $\left(x_{n}\right)$.

Definition 3. Let $X, Y$ be Banach spaces and $T \in L(X, Y)$. Define

$$
\Psi(T)=\sup \left\{\psi\left(T x_{n}\right): x_{n} \stackrel{w}{\rightarrow} 0,\left\|x_{n}\right\| \leq 1\right\} .
$$

Clearly, if $T \in L(X, Y)$ and $x_{n} \stackrel{w}{\rightarrow} 0$ in $X$, then $T x_{n} \stackrel{w}{\rightarrow} 0$ in $Y$. Thus, by [12, Theorem 2], $\Psi(T)=0$ if and only if $T$ has the WBS property. Applying Proposition 2, we can show, as in the proof of Proposition 2.5 of [7], that $\Psi$ is a seminorm in $L(X, Y)$. The procedure of stabilization of $\psi$ plays a key role also in the next result. The arguments of the proof are similar to those used in the proofs of Theorem 3 of [12] and Theorem 3.2 of [7].

Theorem 4. Let $\left(X_{\nu}\right)$ and $\left(Y_{\nu}\right)$ be sequences of Banach spaces and let $\left(T_{\nu}\right)$ be a sequence of operators such that $T_{\nu} \in \mathcal{L}\left(X_{\nu}, Y_{\nu}\right)$ for every $\nu \in \mathbb{N}$ and $\sup _{\nu \in \mathbb{N}}\left\|T_{\nu}\right\|<\infty$. If a Banach sequence lattice $E$ has the $B S$ property and $T \in L\left(E\left(X_{\nu}\right), E\left(Y_{\nu}\right)\right)$ is given by $T x=\left(T_{\nu} x(\nu)\right)$ for every $x=(x(\nu)) \in$ $E\left(X_{\nu}\right)$, then $\Psi(T)=\sup _{\nu \in \mathbb{N}} \Psi\left(T_{\nu}\right)$.

Proof. It is enough to prove that $\Psi(T) \leq \sup _{\nu \in \mathbb{N}} \Psi\left(T_{\nu}\right)$, since $E\left(X_{\nu}\right)$ and $E\left(Y_{\nu}\right)$ contain isometric copies respectively of $X_{\nu}$ and $Y_{\nu}$. Let us fix $\varepsilon>0$ and choose a weakly null sequence $\left(x_{n}\right)$ in the unit ball of $E\left(X_{\nu}\right)$ so that $\Psi(T)-\varepsilon \leq \psi\left(T x_{n}\right)$.

First, we show that we can focus on a finite number of coordinates of the direct sums. Let $t_{n}=\left(\left\|T_{\nu} x_{n}(\nu)\right\|_{Y_{\nu}}\right)$ for every $x_{n}=\left(x_{n}(\nu)\right)$. Since $E$ has the BS property, passing to a subsequence, we may assume that the Cesàro means of all subsequences of $\left(t_{n}\right) \subset E$ converge to the same limit $t \in E$ (see [4]). Then $\psi\left(t_{n}^{0}-t\right)=0$ for every sequence $\left(t_{n}^{0}\right)$ of sam for $\left(t_{n}\right)$ and, by Proposition $2,\left(t_{n}^{0}\right)$ can be taken so that for every finite $A \subset \mathbb{N}$,

$$
\left\||A|^{-1} \sum_{n \in A} t_{n}^{0}-t\right\|_{E}<\frac{\varepsilon}{2} .
$$

Let $\left(I_{n}\right)$ be a sequence of finite subsets of $\mathbb{N}$ with $\left|I_{n}\right|=m$ and $\max I_{n}<$ $\min I_{n+1}$ for all $n$ such that $t_{n}^{0}=m^{-1} \sum_{i \in I_{n}} t_{i}$. Put $x_{n}^{0}=m^{-1} \sum_{i \in I_{n}} x_{i}$. 
For every $r \in \mathbb{N}$ and $z=(z(\nu))$, we will write $P_{r} z=(z(1), \ldots, z(r), 0,0, \ldots)$ and $Q_{r} z=z-P_{r} z$. Since the reflexive lattice $E$ is $\sigma$-order continuous, there is $r \in \mathbb{N}$ such that $\left\|Q_{r} t\right\|_{E}<\varepsilon / 2$. It follows that

$$
\left\|Q_{r}\left(|A|^{-1} \sum_{n \in A} t_{n}^{0}\right)\right\|_{E}<\frac{\varepsilon}{2}+\left\|Q_{r} t\right\|_{E}<\varepsilon .
$$

Thus, for every finite $A \subset \mathbb{N}$,

$$
\begin{aligned}
\varepsilon & >\left\|Q_{r}\left(|A|^{-1} \sum_{n \in A} t_{n}^{0}\right)\right\|_{E}=\left\|Q_{r}\left(|A|^{-1} \sum_{n \in A} \frac{1}{m} \sum_{i \in I_{n}}\left\|T_{\nu} x_{i}(\nu)\right\|_{Y_{\nu}}\right)\right\|_{E} \\
& \geq\left\|Q_{r}\left(|A|^{-1} \sum_{n \in A}\left\|T_{\nu} x_{n}^{0}(\nu)\right\|_{Y_{\nu}}\right)\right\|_{E} \geq\left\|Q_{r}\left(\left\||A|^{-1} \sum_{n \in A} T_{\nu} x_{n}^{0}(\nu)\right\|_{Y_{\nu}}\right)\right\|_{E} \\
& =\left\||A|^{-1} \sum_{n \in A} Q_{r} T x_{n}^{0}\right\|_{E\left(Y_{\nu}\right)} .
\end{aligned}
$$

Passing to a subsequence of $\left(x_{n}^{0}\right)$, we may assume that for each coordinate $1 \leq \nu \leq r$ the limit $\lambda_{\nu}=\lim _{n}\left\|x_{n}^{0}(\nu)\right\|$ exists and $\left\|x_{n}^{0}(\nu)\right\|<\lambda_{\nu}+\varepsilon /\left\|P_{r} e\right\|_{E}$ for every $n$, where $e=(1,1, \ldots)$. Put $\alpha_{\nu}=\lambda_{\nu}+\varepsilon /\left\|P_{r} e\right\|_{E}$. By the equipollence of blocks, all sequences of sam for $\left(x_{n}\right)$ are weakly null and, by Lemma 1 , so are all sequences restricted to coordinates. Now we stabilize $\psi$ consecutively on coordinates $k=1,2, \ldots, r$. Write $y_{n}^{0}(\nu)=T_{\nu} x_{n}^{0}(\nu) / \alpha_{\nu}$.

In the first step, we apply Proposition 2 to $\left(y_{n}^{0}(1)\right)$. There is a sequence $\left(x_{n}^{1}\right)$ of sam for $\left(x_{n}^{0}\right)$ such that for the sequence $\left(y_{n}^{1}(1)\right)$ of sam for $\left(y_{n}^{0}(1)\right)$, where $y_{n}^{1}(1)=T_{1} x_{n}^{1}(1) / \alpha_{1}$, we have

$$
\left\||A|^{-1} \sum_{n \in A} y_{n}^{1}(1)\right\|_{Y_{1}} \leq \psi\left(y_{n}^{1}(1)\right)+\varepsilon
$$

for all finite $A \subset \mathbb{N}$. We put $y_{n}^{1}(\nu)=T_{\nu} x_{n}^{1}(\nu) / \alpha_{\nu}$ for $\nu \neq 1$.

Let $k>1$. By Proposition 2 applied to $\left(y_{n}^{k-1}(k)\right)$, we obtain a sequence $\left(x_{n}^{k}\right)$ of sam for $\left(x_{n}^{k-1}\right)$ such that for the sequence $\left(y_{n}^{k}(k)\right)$ of sam for $\left(y_{n}^{k-1}(k)\right)$, where $y_{n}^{k}(k)=T_{k} x_{n}^{k}(k) / \alpha_{k}$, we have

$$
\left\||A|^{-1} \sum_{n \in A} y_{n}^{k}(k)\right\|_{Y_{k}} \leq \psi\left(y_{n}^{k}(k)\right)+\varepsilon
$$

for all finite $A \subset \mathbb{N}$. Again we put $y_{n}^{k}(\nu)=T_{\nu} x_{n}^{k}(\nu) / \alpha_{\nu}$ for $\nu \neq k$. Since the relation sam is transitive, all sequences $\left(y_{n}^{r}(\nu)\right), 1 \leq \nu \leq r$, are built on the common sequence $\left(x_{n}^{r}\right)$ of sam for $\left(x_{n}^{\nu}\right)$. Consequently,

$\left\||A|^{-1} \sum_{n \in A} y_{n}^{r}(\nu)\right\|_{Y_{\nu}} \leq \psi\left(y_{n}^{\nu}(\nu)\right)+\varepsilon \leq \psi\left(y_{n}^{\nu+1}(\nu)\right)+\varepsilon \leq \cdots \leq \psi\left(y_{n}^{r}(\nu)\right)+\varepsilon$ 
for all finite $A \subset \mathbb{N}$ and every $1 \leq \nu \leq r$. Clearly, $\left\|x_{n}^{r}(\nu) / \alpha_{\nu}\right\|_{X_{\nu}} \leq 1$ for all $n$. It follows that

$$
\begin{aligned}
& \left\||A|^{-1} \sum_{n \in A} P_{r} T x_{n}^{r}\right\|_{E\left(Y_{\nu}\right)}=\left\|P_{r}\left(\alpha_{\nu}\left\||A|^{-1} \sum_{n \in A} y_{n}^{r}(\nu)\right\|_{Y_{\nu}}\right)\right\|_{E} \\
& \leq\left\|P_{r}\left(\lambda_{\nu}+\varepsilon /\left\|P_{r} e\right\|_{E}\right)\right\|_{E} \max _{1 \leq \nu \leq r}\left\||A|^{-1} \sum_{n \in A} y_{n}^{r}(\nu)\right\|_{Y_{\nu}} \\
& \leq(1+\varepsilon)\left(\max _{1 \leq \nu \leq r} \psi\left(y_{n}^{r}(\nu)\right)+\varepsilon\right) .
\end{aligned}
$$

Assume that $\max _{1 \leq \nu \leq r} \psi\left(y_{n}^{r}(\nu)\right)$ is attained for $j, 1 \leq j \leq r$. By transitivity of the relation sam, $\left(x_{n}^{r}\right)$ is a sequence of sam for $\left(x_{n}\right)$. It follows that

$$
\begin{aligned}
\Psi(T)-\varepsilon & \leq \psi\left(T x_{n}\right) \leq \psi\left(T x_{n}^{r}\right) \leq\left\||A|^{-1} \sum_{n \in A} T x_{n}^{r}\right\|_{E\left(Y_{\nu}\right)} \\
& \leq\left\||A|^{-1} \sum_{n \in A} P_{r} T x_{n}^{r}\right\|_{E\left(Y_{\nu}\right)}+\left\||A|^{-1} \sum_{n \in A} Q_{r} T x_{n}^{r}\right\|_{E\left(Y_{\nu}\right)} \\
& \leq(1+\varepsilon)\left(\psi\left(y_{n}^{r}(j)\right)+\varepsilon\right)+\varepsilon \leq(1+\varepsilon)\left(\Psi\left(T_{j}\right)+\varepsilon\right)+\varepsilon .
\end{aligned}
$$

Since $\varepsilon>0$ was chosen arbitrary, $\Psi(T) \leq \sup _{\nu \in \mathbb{N}} \Psi\left(T_{\nu}\right)$.

Considering the identity operator on $E\left(X_{\nu}\right)$, we obtain the following corollary which includes Partington's [12] qualitative result. By an example of [12], the BS property of $E$ cannot be replaced here by the WBS property.

Corollary 5. Let $E$ have the $B S$ property. Then $E\left(X_{\nu}\right)$ has the WBS property if and only if every $X_{\nu}$ has the WBS property.

\section{REFERENCES}

[1] Banach, S., Saks, S., Sur la convergence forte dans les champs $L^{p}$, Studia Math. 2 (1930), 51-57.

[2] Beauzamy, B., Banach-Saks properties and spreading models, Math. Scand. 44 (1979), 357-384.

[3] Brunel, A., Sucheston, L., On B-convex Banach spaces, Math. Systems Theory 7 (1974), 294-299.

[4] Erdös, P., Magidor, M., A note on regular methods of summability and the Banach-Saks property, Proc. Amer. Math. Soc. 59 (1976), 232-234.

[5] Krassowska, D., Płuciennik, R., A note on property $(H)$ in Köthe-Bochner sequence spaces, Math. Japon. 46 (1997), 407-412.

[6] Krein, S. G., Petunin, Yu. I., Semenov, E. M., Interpolation of linear operators, Translations of Mathematical Monographs, 54. American Mathematical Society, Providence, R.I., 1982.

[7] Kryczka, A., Alternate signs Banach-Saks property and real interpolation of operators, Proc. Amer. Math. Soc. 136 (2008), 3529-3537. 
[8] Kryczka, A., Mean separations in Banach spaces under abstract interpolation and extrapolation, J. Math. Anal. Appl. 407 (2013), 281-289.

[9] Lin, P.-K., Köthe-Bochner function spaces, Birkhäuser Boston, Inc., Boston, MA, 2004

[10] Lindenstrauss, J., Tzafriri, L., Classical Banach spaces. II. Function spaces, SpringerVerlag, Berlin-New York, 1979.

[11] Mastyło, M., Interpolation spaces not containing $l_{1}$, J. Math. Pures Appl. 68 (1989), 153-162.

[12] Partington, J. R., On the Banach-Saks property, Math. Proc. Cambridge Philos. Soc. 82 (1977), 369-374.

[13] Rosenthal, H. P., Weakly independent sequences and the Banach-Saks property, Bull. London Math. Soc. 8 (1976), 22-24.

[14] Szlenk, W., Sur les suites faiblement convergentes dans l'espace L, Studia Math. 25 (1965), 337-341.

Andrzej Kryczka

Institute of Mathematics

Maria Curie-Skłodowska University

20-031 Lublin

Poland

e-mail: andrzej.kryczka@umcs.pl

Received November 5, 2013 